DENV-3, este hallazgo significó el reingreso a Loreto de este serotipo y la circulación del mismo en la trifrontera. Por ahora, ninguna muestra resultó positiva para CHIKV.

La isla Santa Rosa y la ciudad de Caballococha son lugares estratégicos, no solo para vigilancia epidemiológica, sino también para realizar vigilancia entomológica como la detección de Aedes albopictus en Leticia ${ }^{(4)}$. En la trifrontera existe una población de más de cien mil personas; mayoritariamente integrantes de la etnia tikuna y población itinerante (migrantes, comerciantes, etc. $)^{(5)}$. Es una puerta de entrada de enfermedades al país, por la estrecha actividad económica que determina un alto movimiento de personas entre las ciudades fronterizas (Caballococha, Leticia y Tabatinga) que comparten características epidemiológicas importantes, sin mencionar la presencia de CHIKV y DENV en el departamento fronterizo de Amazonas en Colombia ${ }^{(6)}$.

Por lo tanto, es importante que el MINSA considere reforzar y reimplementar la vigilancia centinela en esta zona fronteriza, no solo para dengue y chikungunya, sino para otros arbovirus (mayaro, una, zika, encefalitis equina venezolana, oropuche, etc.) además de otros patógenos de importancia. Esto nos permitirá estar atentos y preparados ante la introducción o reintroducción de enfermedades que pueden generar daño a la salud pública.

Descargo de responsabilidad: las opiniones y afirmaciones contenidas aquí son propias de los autores y no deben interpretarse como posición oficial o que reflejen la opinión del Ministerio de Salud del Perú.

Fuentes de financiamiento: para la presente carta al editor no se contó con ninguna fuente de financiamiento.

Conflictos de interés: los autores declaran no tener conflictos de interés en la publicación de esta carta.

\section{REFERENCIA BIBLIOGRÁFICAS}

1. Organización Mundial de la Salud. Reglamento Sanitario Internacional; $2^{a}$ ed. Ginebra: WHO; 2005.

2. Equipo Tecnico Perú-Colombia. Analisis de la Situación de Salud de Fronteras Putumayo/Amazonas/Loreto. ColombiaPerú. 2014.

3. Instituto Nacional de Estadistica e Informatica [Internet]. Lima, Perú: INEI; c2015 [citado el 10 de setiembre del 2015]. Disponible en: http://www.inei.gob.pe/estadisticas/indicetematico/population-estimates-and-projections/

4. Velez ID, Quiñones ML, Suárez M, Olano V, Murcia LM, Correa E, et al. Presencia de Aedes albopictus en Leticia, Amazonas, Colombia. Biomedica. 1998;18(3):192-8.

5. Suárez-Mutis MC, Mora C, Perez L, Peiter PC. Interacciones transfronterizas y salud en la frontera de Brasil-Colombia-
Perú. Mundo Amazonico. 2010;1:243-66. DOI: http:// dx.doi.org/10.5113/ma.1.10268

6. Instituto Nacional de Salud de Colombia. Comportamiento de los eventos de vigilancia en salud pública Enfermedades transmitidas por vectores [Internet]. Boletin Epidemiologico Semanal. 2015;(32):20-9 [citado el 10 de setiembre del 2015]. Disponible en: http://www.ins.gov.co/boletinepidemiologico/Boletn \%20Epidemiolgico/2015\%20 Boletin\%20epidemiologico\%20semana\%2032.pdf

Correspondencia: Erik Jair Jhonston Vela

Dirección: Calle Napo 1298. Iquitos, Perú.

Teléfono: (065) 972838825

Correoelectrónico:erik_jhonston@hotmail.com

\section{INCREMENTO EN EL GASTO POR INVERSIÓN EN EL SECTOR SALUD: ¿EFICIENCIA Y EFECTIVIDAD EN EL GASTO?}

\section{INCREASED SPENDING FROM INVESTMENT IN THE HEALTH SECTOR: EFFICIENCY AND COST EFFECTIVENES IN SPENDING?}

\author{
Carlos A. Huayanay-Espinoza ${ }^{1}$, Andrés Huayanay ${ }^{2,3}$, \\ Luis Huicho ${ }^{1,4,5,6}$
}

Sr. Editor. El Ministerio de Economía y Finanzas define a un proyecto de inversión pública (PIP) como toda intervención limitada en el tiempo, que utiliza total o parcialmente recursos públicos, con el fin de crear, ampliar, mejorar, modernizar o recuperar la capacidad productora de bienes o servicios, cuyos beneficios se generen durante la vida útil del proyecto y sean independientes de los de otros proyectos ${ }^{(1)}$. La implementación de políticas tales como la descentralización ${ }^{(3)}$ y el uso de instrumentos como el presupuesto por resultados ${ }^{(4)}$ han ido de la mano con cambios notables en la estructura del gasto de gobierno a través del tiempo.

\footnotetext{
Centro de Investigación para el Desarrollo Integral y Sostenible (CIDIS), Universidad Peruana Cayetano Heredia.

2 Dirección General de Administración, Universidad Nacional San Cristóbal de Huamanga.

3 Escuela de Formación Profesional de Ingeniera Comercial y Negocios Internacionales, Universidad de Ayacucho Federico Froebel.

4 Facultad de Medicina, Universidad Peruana Cayetano Heredia

5 Universidad Nacional Mayor de San Marcos.

6 Instituto Nacional de Salud del Niño. Lima, Perú. Recibido: 25-07-15 Aprobado: 05-08-15
}

Citar como: Huayanay-Espinoza CA, Huayanay A, Huicho L. Incremento en el gasto por inversión en el sector salud: ¿eficiencia y efectividad en el gasto? [carta]. Rev Peru Med Exp Salud Publica. 2015;32(4):822-4. 

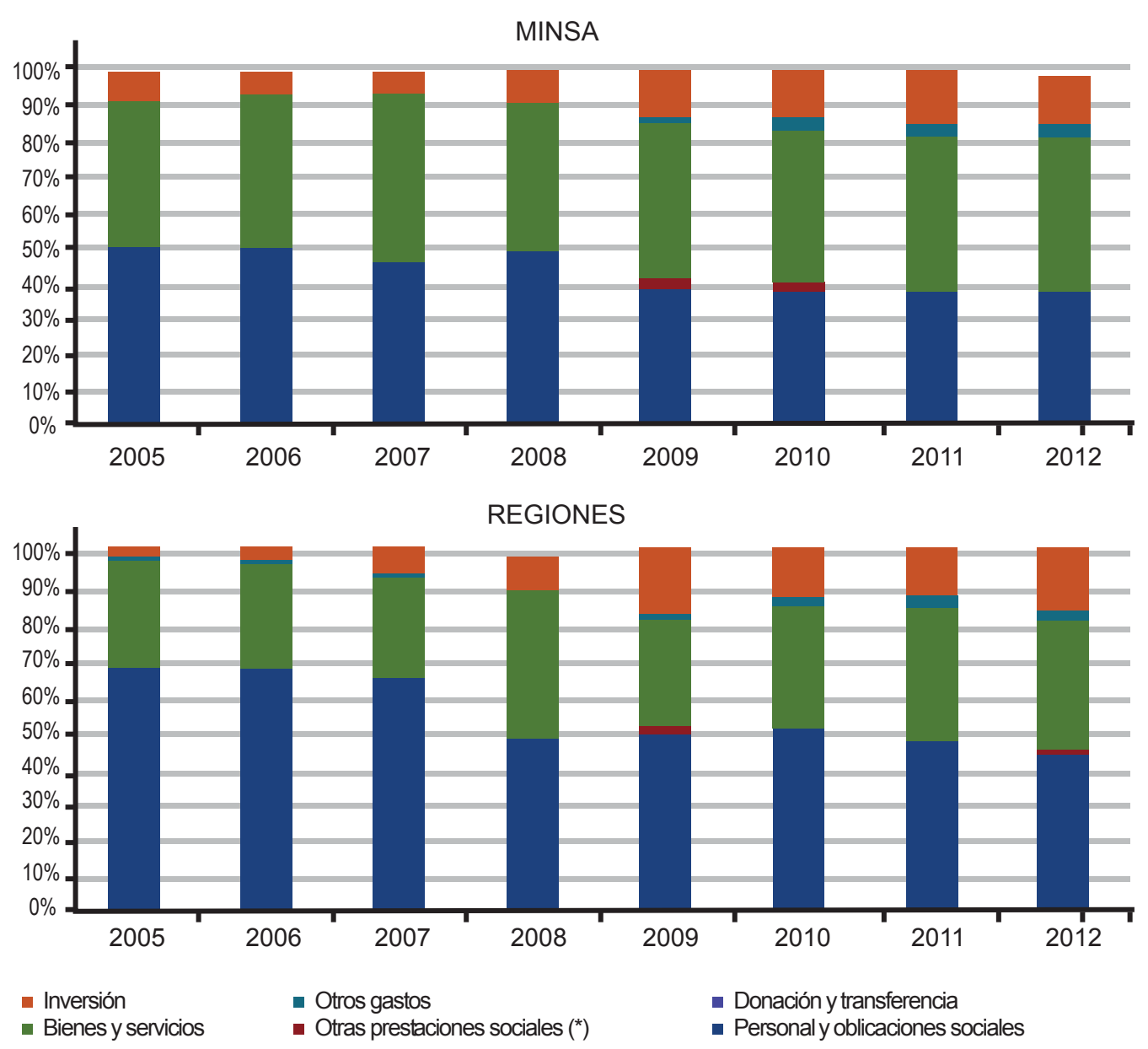

Figura 1. Estructura del gasto de gobierno en salud a nivel del MINSA y de las regiones

Fuente: Cuentas Nacionales de Salud, Perú:1995-2012(2).

Dentro de este contexto, cobra relevancia hacer un breve análisis del gasto en salud en nuestro país. Según el último estudio de Cuentas Nacionales en Salud, el gasto per cápita en salud se incrementó de 419,2 soles en el 2005 a 873,1 soles el 2012 (2). Sin embargo, este incremento debe examinarse dentro del marco más amplio de la evolución en la estructura del gasto. La Figura 1 muestra dicha estructura, tanto en el Ministerio de Salud (MINSA) como en las regiones.

El porcentaje de gasto total del sector Salud en personal y obligaciones sociales pasó del 57\% el año 2005 al $41 \%$ el año 2012, reducción que fue proporcionarlmente similar tanto para el MINSA como a nivel regional. Por otro lado, el gasto ejecutado en inversión (PIP) en el MINSA se incrementó de $8,4 \%$ el 2005 a $14,0 \%$ el 2012 , mientras que a nivel de las regiones se incrementó de $2,4 \%$ el 2005 a $19,1 \%$ el 2012 (Figura 1).
Estos cambios en la estructura de la ejecución del gasto muestran la importancia creciente que se le ha dado a los PIP. Sin embargo, esto solo es parte de la historia que debe ser analizada. Gastar más en PIP no significa necesariamente gastar mejor. Para tener el panorama completo, se necesita analizar si los PIP culminaron satisfactoriamente y se tradujeron, por ejemplo, en nuevos establecimientos de salud o educativos plenamente operativos, con infraestructura, equipos y recursos humanos adecuados. Más aun, se necesita evaluar si los PIP se ejecutaron en las zonas de mayor necesidad.

Por otra parte, la evolución del gasto en el sector salud debe ser relacionada con la evolución de los indicadores de cobertura de intervenciones relevantes y con la de indicadores de impacto (como nutrición y mortalidad), de modo que se tenga una idea más precisa de la eficiencia y la efectividad del gasto. Para capturar cuán equitativo fue el gasto, este análisis debe ser desagregado, por 
ejemplo, según ámbitos rural y urbano y según quintiles de pobreza ${ }^{(5)}$. Estos aspectos deben ser prioritarios en la discusión sobre financiamiento en salud, de modo que se formulen presupuestos más eficientes y efectivos, con un enfoque de equidad.

\section{REFERENCIAS BIBLIOGRÁFICAS}

1. Ministerio de Economía y Finanzas del Perú. Glosario oficial del Ministerio de Economía y Finanzas [Internet]. Lima: MEF; c2015 [citado el 23 de Julio de 2015]. Disponible en: $\quad$ https://www.mef.gob.pe/index.php?option $=$ com glossary\&letter = P \&id = 383\&lang=eshttps:// www.mef.gob.pe/index.php?option = com glossary\&letter $=$ P\&id=383\&lang=es

2. Ministerio de Salud del Perú, Organización Panamericana de la Salud (OPS). Cuentas Nacionales en Salud, Perú: 19952012. Lima: OPS/OMS; 2015.

3. La Contraloría General de la República. Estudio del proceso de descentralización en el Perú. Lima: La contraloría General de la República; 2014.
4. Acuña R, Huaita F, Mesinas J. Un camino de un presupuesto por resultados (PpR): Una nota sobre los avances recientes en la programación presupuestaria. [Internet]. Lima: Ministerio de Economía y Finanzas; 2012 [citado el 17 noviembre de 2015]. Disponible en: http://gestionensalud.medicina. unmsm.edu.pe/wp-content/uploads/2015/08/PM_RB_01_ En_caminode_un_PpR.pdf

5. Victora CG, Barros AJ, Axelson H, Bhutta ZA, Chopra M, França GV, et al. How changes in coverage affect equity in maternal and child health interventions in 35 Countdown to 2015 countries: an analysis of national surveys. Lancet. 2012;29;380(9848):1149-56. doi: 10.1016/S01406736(12)61427-5.

Correspondencia: Carlos A. Huayanay Espinoza

Dirección: Av. Honorio Delgado s/n. Universidad Peruana Cayetano Heredia.

Teléfono: (511) 997915901

Correo electrónico: carlos.huayanay@upch.pe
REVISTA PERUANA DE MEDICINA EXPERIMENTAL Y SALUD PÚBLICA CUMPLIENDO SUS METAS Y PROYECTÁNDOSE AL FUTURO

\footnotetext{
Visite los contenidos de la revista en: www.ins.gob.pe/rpmesp
} 